\title{
Topological type of discriminants of some special families
}

\author{
Evelia R. García Barroso ${ }^{1}$ M. Fernando Hernández Iglesias ${ }^{2,3}$
}

Accepted: 20 November 2020 / Published online: 8 September 2021

(c) The Author(s) 2021

\begin{abstract}
We will describe the topological type of the discriminant curve of the morphism $(\ell, f)$, where $\ell$ is a smooth curve and $f$ is an irreducible curve (branch) of multiplicity less than five or a branch such that the difference between its Milnor number and Tjurina number is less than 3 . We prove that for a branch of these families, the topological type of the discriminant curve is determined by the semigroup, the Zariski invariant and at most two other analytical invariants of the branch.
\end{abstract}

Keywords Discriminant curve $\cdot$ Nondegenerate singularity $\cdot$ Newton polygon $\cdot$ Zariski invariant $\cdot$ Milnor number $\cdot$ Tjurina number

Mathematics Subject Classification Primary $14 \mathrm{~J} 17 \cdot$ Secondary 32S15

\section{Introduction}

Let $f(x, y) \in \mathbb{C}\{x, y\}$ be irreducible. The germ of the irreducible analytic curve (branch) of equation $f(x, y)=0$ is denoted by $C \equiv f(x, y)=0$. Observe that the curves $f(x, y)=0$ and $u(x, y) f(x, y)=0$ are the same, for any unit $u(x, y) \in \mathbb{C}\{x, y\}$. The multiplicity of $C$, denoted by $m(C)$, is by definition the order of the power series $f(x, y)$. Suppose that $C$ has multiplicity $n$. We will say that $C$ is singular if $n>1$. Otherwise $C$ is a smooth curve. The initial form of $f(x, y)$ is the sum of all terms of $f(x, y)$ of degree $n$. Since $f$ is irreducible, its initial form is a power of a linear form. After a linear change of coordinates, if necessary,

Evelia R. García Barroso was partially supported by the Spanish Project MICINN PID2019-105896GB-I00.

$\bigotimes$ Evelia R. García Barroso ergarcia@ull.es

M. Fernando Hernández Iglesias

mhernandezi@unmsm.edu.pe ; mhernandezi@pucp.pe

1 Dpto. Matemáticas, Estadística e I.O. Sección de Matemáticas, Universidad de La Laguna, Apartado de Correos 456, 38200 La Laguna, Tenerife, Spain

2 Facultad de Ciencias Matemáticas, Escuela de Matemática, Universidad Nacional Mayor de San Marcos, Cercado de Lima 15081, Perú

3 Dpto. Ciencias - Sección Matemáticas, Pontificia Universidad Católica del Perú, Av. Universitaria, Lima 32, San Miguel 1801, Perú 
we can suppose that the initial form of $f(x, y)$ is $y^{n}$. Suppose that $C$ has multiplicity $n>1$. We denote by $\mathbb{N}^{*}$ the set of positive integers. By Newton's theorem [7, Theorem 3.8] there is $\alpha\left(x^{1 / n}\right) \in \mathbb{C}\{x\}^{*}=\bigcup_{m \in \mathbb{N}^{*}} \mathbb{C}\left\{x^{1 / m}\right\}$ with $\alpha(0)=0$ such that $f\left(x, \alpha\left(x^{1 / n}\right)\right)=0$ and we say that $\alpha\left(x^{1 / n}\right) \in \mathbb{C}\{x\}^{*}$ is a Newton-Puiseux root of $C$. Let us denote by $\operatorname{Zer}(f)$ the set of Newton-Puiseux roots of $C$. Let $\alpha\left(x^{1 / n}\right) \in \operatorname{Zer}(f)$. After Puiseux's theorem [7, Corollary 3.12] we have that $\operatorname{Zer}(f)=\left\{\alpha_{j}:=\alpha\left(\omega^{j} x^{1 / n}\right)\right\}_{j=1}^{n}$, where $\omega$ is an $n$ th-primitive root of unity. Hence

$$
f(x, y)=u(x, y) \prod_{j=1}^{n}\left(y-\left(\alpha\left(\omega^{j} x^{1 / n}\right)\right)\right),
$$

where $u \in \mathbb{C}\{x, y\}$ is a unit. After a change of coordinates, if necessary, we can write $\alpha(x)=\sum_{i \geq s_{1}} a_{i} x^{i / n}$, where $s_{1}>n$ and $s_{1} \not \equiv 0 \bmod n$.

If we put $x=t^{n}$, where $t$ is a new variable, the Newton-Puiseux root $\alpha\left(x^{1 / n}\right)$ can be written as

$$
\left\{\begin{array}{l}
x(t)=t^{n} \\
y(t)=\sum_{i \geq s_{1}} a_{i} t^{i}
\end{array}\right.
$$

that we will call the Puiseux parametrisation of $C$.

There are $g \in \mathbb{N}$ and a sequence $\left(\beta_{0}=n<\beta_{1}=s_{1}<\beta_{2}<\cdots<\beta_{g}\right)$ of nonnegative integers such that

$$
\left\{\operatorname{ord}\left(\alpha_{i}-\alpha_{j}\right): \alpha_{i}, \alpha_{j} \in \operatorname{Zer}(f), i \neq j\right\}=\left\{\frac{\beta_{l}}{\beta_{0}}: 1 \leq l \leq g\right\} \subseteq \mathbb{Q} \backslash \mathbb{Z} .
$$

The sequence $\left(\beta_{0}, \cdots, \beta_{g}\right) \subseteq \mathbb{N}$ is called the sequence of characteristic exponents of $C$. The number $g$ is a topological invariant called the genus of the branch $C$.

Consider the set

$$
S(C):=\left\{i_{0}(f, h): h \in \mathbb{C}\{x, y\}, h \neq \equiv \bmod f\right\},
$$

where $i_{0}(f, h)=\operatorname{dim}_{\mathbb{C}} \mathbb{C}\{x, y\} /(f, h)$ is the intersection number (or intersection multiplicity) of $f(x, y)=0$ and $h(x, y)=0$ at the origin. It is well known that $S(C)$ is a semigroup called the semigroup of values of the branch $C$. The complement of $S(C)$ in $\mathbb{N}$ is finite. The conductor of $S(C)$ is by definition the smallest natural number $c \in \mathbb{N}$ such that every natural number $N \in \mathbb{N}$ with $N \geq c$ is an element of $S(C)$.

The semigroup $S(C)$ admits a minimal system of generators $\left(s_{0}, s_{1}, \ldots, s_{g}\right)$, where $s_{i-1}<$ $s_{i}, g$ is the genus of $C, s_{0}=n=i_{0}(f, x)$ and $s_{1}=m=: i_{0}(f, y)$. It is a well-known property of $S(C)\left[7\right.$, p. 88, (6.5)] that $e_{k}:=\operatorname{gcd}\left(s_{0}, \ldots, s_{k}\right)=\operatorname{gcd}\left(\beta_{0}, \ldots, \beta_{k}\right)$ for $0 \leq k \leq g$ and $e_{k-1} s_{k}<e_{k} s_{k+1}$ for $1 \leq k \leq g-1$.

If $n>2$, then we have $c \geq s_{1}+1$. Let $q$ be the number of natural numbers between $s_{1}$ and $c$ which are not in $S(C)$. We can verify (see [16, p. 21]) that $q=\frac{c}{2}-s_{1}+\left[\frac{s_{1}}{s_{0}}\right]+1$, for $s_{0}=n>2$, where $[z]$ denotes the integral part of $z \in \mathbb{R}$.

Let $f, h \in \mathbb{C}\{x, y\}$ be irreducible power series. Using the Halphen-Zeuthen formula we get

$$
i_{0}(f, h)=\sum_{i, j} \operatorname{ord}\left(\gamma_{j}-\alpha_{i}\right)
$$

where Zer $f=\left\{\alpha_{i}\right\}_{i}$ and Zer $h=\left\{\gamma_{j}\right\}_{j}$.

Two branches $C$ and $D$ have the same topological type (or they are equisingular) if they are topologically equivalent as embedded curves in $\mathbb{C}^{2}$. It is well known [16, Chapter II] 
that two branches are equisingular if and only if they have the same semigroup of values or equivalently they have the same characteristic exponents. Denote by $\mathcal{E}(C)$ the set of branches which are equisingular to $C$. In the set $\mathcal{E}(C)$ we define the next equivalence relation: two branches $D_{1}$ and $D_{2}$ in $\mathcal{E}(C)$ are analytically equivalent, and we will denote this by $D_{1} \cong D_{2}$, if there exists an analytic isomorphism $T: U_{1} \longrightarrow U_{2}$ such that $U_{i}$ are neighbourhoods of the origin, $D_{i}$ is defined in $U_{i}, 1 \leq i \leq 2$ and $T\left(D_{1} \cap U_{1}\right)=D_{2} \cap U_{2}$.

The moduli space of the equisingularity class $\mathcal{E}(C)$ is the quotient space $\mathcal{E}(C) / \cong$. Let $v_{1}<v_{2}<\cdots<v_{q}$ be the integers of the set $\left\{s_{1}+1, \ldots, c-1\right\}$ which are not in $S(C)$. Zariski proved [16, Proposition 1.2, Chapter III] that there exists a branch $\bar{C}$ analytically equivalent to $C$, parametrized as follows:

$$
\left\{\begin{array}{l}
\bar{x}=t^{n} \\
\bar{y}=t^{s_{1}}+\sum_{i=1}^{q} a_{i} t^{v_{i}}
\end{array}\right.
$$

Put $\Omega:=\{\omega=g(x, y) d x+h(x, y) d y: g, h \in \mathbb{C}\{x, y\}\}$. If $(x(t), y(t))$ is a Puiseux parametrisation of $C$, we put

$$
v(\omega):=\operatorname{ord}\left(f(x(t), y(t)) x^{\prime}(t)+g(x(t), y(t)) y^{\prime}(t)\right)+1 .
$$

Let $\Lambda:=\{v(\omega): \omega \in \Omega\}$. If $\Lambda \backslash S(C) \neq \emptyset$, then the number $\lambda:=\min (\Lambda \backslash S(C))-v_{0}$ is an analytical invariant of $C$ called the Zariski invariant.

Using [16, Lemma 2.6, Chapter IV], we can rewrite the parametrization (1.4) in the next form:

$$
\left\{\begin{array}{l}
\bar{x}=t^{n}, \\
\bar{y}=t^{s_{1}}+a t^{\lambda}+\text { a finite sum of terms } a_{i} t^{\nu_{i}},
\end{array}\right.
$$

where $a \neq 0, v_{i}>\lambda>s_{1}$.

The Normal Forms Theorem (see [8, Theorem 1]) states that the plane branch $C$ with semigroup of values $S(C)=\left\langle s_{0}, \ldots, s_{g}\right\rangle$ and value set of differentials $\Lambda$ is either analytically equivalent to a branch with Puiseux parametrization $\left(t^{s_{0}}, t^{s_{1}}\right)$ or Puiseux parametrization $\left(t^{s_{0}}, t^{s_{1}}+t^{\lambda}+\sum_{i>\lambda, i \notin \Lambda-s_{0}} a_{i} t^{i}\right)$.

Let $f(x, y)=\sum_{i, j} a_{i j} x^{i} y^{j} \in \mathbb{C}\{x, y\}$. The support of $f$ is $\operatorname{supp}(f):=\left\{(i, j) \in \mathbb{N}^{2}\right.$ : $\left.a_{i j} \neq 0\right\}$. The Newton polygon of $f$, denoted by $\mathcal{N}(f)$, is by definition the convex hull of $\operatorname{supp}(f)+\mathbb{R}_{\geq 0}^{2}$. Observe that $\mathcal{N}(f)=\mathcal{N}(u f)$ for any unit $u \in \mathbb{C}\{x, y\}$. Nevertheless, the Newton polygon depends on coordinates. The inclination of any compact face $L$ of $\mathcal{N}(f)$ is by definition the quotient of the length of the projection of $L$ over the horizonal axis by the length of its projection over the vertical axis. The Newton polygon of $f$ gives information on the Newton-Puiseux roots of $f(x, y)=0$. More precisely, if $L$ is a compact face of $\mathcal{N}(f)$ of inclination $i$ and the length of its projection over the vertical axis is $\ell_{2}$, then $f$ has $\ell_{2}$ Newton-Puiseux roots of order $i$ (see [3, Lemme 8.4.2]).

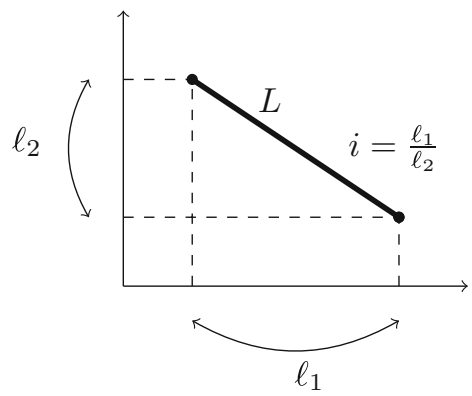


We say that $f(x, y) \in \mathbb{C}\{x, y\}$ is nondegenerate in the sense of Kouchnirenko with respect to the coordinates $(x, y)$, if for any compact edge $L$ of $\mathcal{N}(f)$ the polynomial $f_{L}(x, y):=$ $\sum_{(i, j) \in L \cap \operatorname{supp}(f)} a_{i j} x^{i} y^{j}$ does not have critical points outside the axes $x=0$ and $y=0$, or equivalently, the polynomial $F_{L}(z):=\frac{f_{L}(1, z)}{z^{j_{0}}}$ has no multiple roots, where $j_{0}:=\min \{j \in$ $\mathbb{N}:(i, j) \in L\}$. Since $\mathcal{N}(f)=\mathcal{N}(u f)$, for any unit $u \in \mathbb{C}\{x, y\}$, the notion of nondegeneracy is extended to curves. The topological type of nondegenerate plane curves are completely determined by their Newton polygons (see [13, Proposition 4.7] and [6, Theorem 3.2]).

Let $\ell(x, y)=0$ be a smooth curve and let $f(x, y)=0$ define an isolated singularity at $0 \in \mathbb{C}^{2}$. Assume that $\ell(x, y)$ does not divide $f(x, y)$ and consider the morphism

$$
\begin{aligned}
(\ell, f):\left(\mathbb{C}^{2}, 0\right) & \longrightarrow\left(\mathbb{C}^{2}, 0\right), \\
(x, y) & \longmapsto(u, v):=(\ell(x, y), f(x, y)) .
\end{aligned}
$$

There are two curves associated with $(\ell, f)$ : the polar curve $\frac{\partial \ell}{\partial x} \frac{\partial f}{\partial y}-\frac{\partial \ell}{\partial y} \frac{\partial f}{\partial x}=0$ and its direct image $D(u, v)=0$ which is called the discriminant curve of the morphism $(\ell, f)$.

The topological type of the polar curve depends on the analytical type of $\ell(x, y)=0$ and $f(x, y)=0$. In [9] the authors completely determine the topological type of the generic polar curve when the multiplicity of $f(x, y)=0$ is less than 5 .

The Newton polygon of $D(u, v)$ in the coordinates $(u, v)$ is called the jacobian Newton polygon of the morphism $(\ell, f)$. This notion was introduced by Teissier [14], who proved that the inclinations of this jacobian polygon are topological invariants of $(\ell, f)$ called polar invariants. After Merle [12], when $f$ is irreducible with semigroup of values $S(f)=\left\langle s_{0}, s_{1}, \ldots, s_{g}\right\rangle$ then the jacobian Newton polygon of $(\ell, f)$ has $g$ compact edges $\left\{E_{i}\right\}_{i=1}^{g}$. The length of the projection of $E_{i}$ on the vertical axis is $\left(\frac{e_{i-1}}{e_{i}}-1\right) \cdot \frac{e_{i-1}}{e_{0}}$. The length of the projection of $E_{i}$ on the horizontal axis is $\left(\frac{e_{i-1}}{e_{i}}-1\right) \cdot s_{i}$. Hence the inclinations (quotient between the length of the horizontal projection and the length of the vertical projection) of the compact edges of the jacobian polygon are $s_{1}<\frac{e_{1}}{e_{0}} s_{2}<\frac{e_{2}}{e_{0}} s_{3}<\cdots<\frac{e_{g-1}}{e_{0}} s_{g}$.

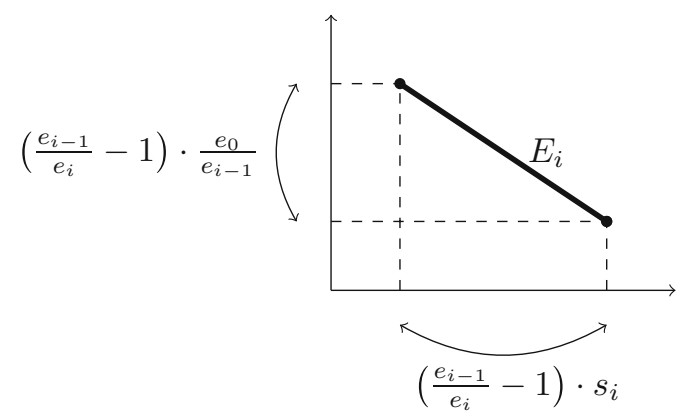

In [5] the authors study the pairs $(\ell, f)$ for which the discriminant curve is nondegenerate in the Kouchnirenko sense. In particular, when $f$ is irreducible, after [5, Corollary 4.4] the discriminant curve $D(u, v)=0$ is nondegenerate if and only if the multiplicity of $f(x, y)=0$ equals 2 or equals 4 and the genus equals 2 . Otherwise the discriminant curve is degenerate. Our aim in this paper will be to describe the topological type of the discriminant curve $D(u, v)=0$ of the morphism $(\ell, f)$, where $f$ is irreducible and belongs to some special families, as for example, branches of multiplicity less than 5 , branches $C$ such that the difference between its Milnor number $\mu(C)$ and Tjurina number $\tau(C)$ is less than 3 , with $\mu(C)=i_{0}\left(\frac{\partial f}{\partial x}, \frac{\partial f}{\partial y}\right)=\operatorname{dim}, \operatorname{not} \operatorname{im}_{\mathbb{C}} \mathbb{C}\{x, y\} /\left(\frac{\partial f}{\partial x}, \frac{\partial f}{\partial y}\right)$ and 
$\tau(C)=\operatorname{dim}_{\mathbb{C}} \mathbb{C}\{x, y\} /\left(f, \frac{\partial f}{\partial x}, \frac{\partial f}{\partial y}\right)$, where $\left(\frac{\partial f}{\partial x}, \frac{\partial f}{\partial y}\right)$ (resp. $\left.\left(\frac{\partial f}{\partial x}, \frac{\partial f}{\partial y}, f\right)\right)$ denotes the ideal of $\mathbb{C}\{x, y\}$ generated by $\frac{\partial f}{\partial x}$ and $\frac{\partial f}{\partial y}$ (resp. by $\frac{\partial f}{\partial x}, \frac{\partial f}{\partial y}$ and $f$ ).

For these families of plane branches we determine the topological type of their discriminant curves, in the spirit of [9]. We prove that the topological type of the discriminant curve $D(u, v)=0$ is determined, at most, by the semigroup of values $S(f)$, the Zariski invariant and two other analytical invariants of the curve $f(x, y)=0$. In all cases we explicitly determine such analytical invariants. Hence, in order to describe the topological type of the discriminant curve of a branch, it is necessarily the same number of analytical invariants of the initial branch, as it happens for its generic polar curves (see [9]). Finally in Sect. 5 we summarize the different topological types of the discriminant curve in some tables.

\section{Equation of the discriminant curve}

An analytic change of coordinates does not affect the discriminant curve of the morphism defined in (1.5) (see for example [2, Section 3]). Hence in what follows we assume that $\ell(x, y)=x$. Then $\frac{\partial f}{\partial y}=0$ is the polar curve of the morphism $(x, f)$.

In this paper we will determine the topological type of the discriminant curve of the morphism (1.5) for $\ell(x, y)=x$ and $f(x, y) \in \mathbb{C}\{x, y\}$ irreducible belonging to some special families. The corresponding study relative to the polar curves was done in [10] and [11], where the authors characterize the equisingularity classes of irreducible plane curve germs whose general members have nondegenerate general polar curves. In addition, they give explicit Zariski open sets of curves in these equisingularity classes whose general polars are nondegenerate and describe their topology.

Suppose that the Newton-Puiseux factorizations of $f(x, y)$ and $\frac{\partial f}{\partial y}(x, y)$ are of the form

$$
\begin{aligned}
f(x, y) & =u_{1}(x, y) \prod_{i=1}^{n}\left[y-\alpha_{i}(x)\right], \\
\frac{\partial f}{\partial y}(x, y) & =u_{2}(x, y) \prod_{j=1}^{n-1}\left[y-\gamma_{j}(x)\right],
\end{aligned}
$$

where $u_{1}(x, y), u_{2}(x, y) \in \mathbb{C}\{x, y\}$ are units, $n=\operatorname{ord} f, \operatorname{Zer}(f)=\left\{\alpha_{i}(x)\right\}_{i}$ and $\operatorname{Zer}\left(\frac{\partial f}{\partial y}\right)=$ $\left\{\gamma_{j}(x)\right\}_{j}$. If $f$ is irreducible of order $n$ then $n$ is the smallest natural number such that $\left\{\alpha_{i}(x)\right\}_{i} \subset \mathbb{C}\left\{x^{1 / n}\right\}$. Moreover, if we fix $\alpha_{i}\left(x^{1 / n}\right)$ then $\alpha_{j}\left(x^{1 / n}\right)=\alpha_{i}\left(\omega x^{1 / n}\right)$ for any $1 \leq j \leq n$, where $w$ is an $n$th root of unity.

Following [4, Lemma 5.4] the discriminant curve of the morphism $(x, f)$ can be written as

$$
D(u, v)=\prod_{j=1}^{n-1}\left(v-f\left(u, \gamma_{j}(u)\right)\right) .
$$

\section{Discriminants of branches of small multiplicities}

In this section we determine the topological type of the discriminant of the morphism given in (1.5), where $C \equiv f(x, y)=0$ has small multiplicity. For this we will make use the results 
of [16] and the analytic classification of plane branches of multiplicity less than or equal to 4 , given in [8].

\subsection{Discriminants of branches of multiplicity 2}

Let $C \equiv f(x, y)=0$ be a branch of multiplicity 2 . The minimal system of generators of the semigroup of $C$ is $\left\langle 2, s_{1}\right\rangle$, where $s_{1}$ is an odd natural number. By [16, Chapitre V] the moduli space of branches of multiplicity 2 have a unique point whose parametrization is given by $\left(t^{2}, t^{s_{1}}\right)$, that is the branch $y^{2}-x^{s_{1}}=0$. Then $f_{y}(x, y)=2 y$ whose Newton-Puiseux root is $y=0$. Hence, after (2.3) we have $D(u, v)=v-f(u, 0)=v+u^{s_{1}}$, that is the discriminant curve is smooth. The Newton polygon of $D(u, v)$ has only one compact edge. The univariate polynomial associated with this edge is $z+1$, so $D(u, v)$ is nondegenerate.

\subsection{Discriminants of branches of multiplicity 3}

Let $C \equiv f(x, y)=0$ be a branch of multiplicity 3 . The minimal system of generators of the semigroup of $C$ is $\left\langle 3, s_{1}\right\rangle$, where $s_{1} \in \mathbb{N}$ is such that $s_{1} \not \equiv 0 \bmod 3$. By [16, Chapitre V] the moduli space of branches of multiplicity 3 is completely determined by the semigroup of the branch and its Zariski invariant $\lambda$. The corresponding normal forms are as follows:

$$
x=t^{3}, \quad y=t^{s_{1}}+a t^{\lambda},
$$

where $a=0$ when $\lambda=0$ or if $\lambda \neq 0$ then we have $a=1$ and

$$
\lambda=\left\{\begin{array}{l}
3 e+3 k+4 \text { when } s_{1}=3 e+2 \\
3 e+3 k+2 \text { when } s_{1}=3 e+1,
\end{array} \text { where } 0 \leq k \leq e-2 .\right.
$$

Observe that if $\lambda \neq 0$ then $\frac{s_{1}+\lambda}{3}$ is a natural number greater than 2 .

Proposition 3.1 Let $C \equiv f(x, y)=0$ be a branch of semigroup $\left\langle 3, s_{1}\right\rangle$ and let the Zariski invariant equal $\lambda$. The discriminant curve $D(u, v)=0$ is degenerate and its topological type is determined by $\left(3, s_{1}, \lambda\right)$ in the following way:

(1) If $\lambda=0$ then the discriminant is the double smooth branch $\left(v+u^{s 1}\right)^{2}=0$.

(2) If $\lambda \neq 0$ then

(a) when $\operatorname{gcd}\left(2, s_{1}+\lambda\right)=2$, the discriminant curve is the union of two smooth branches $D_{i}(u, v)=0,1 \leq i \leq 2$, with intersection number $i_{0}\left(D_{1}, D_{2}\right)=\frac{s_{1}+\lambda}{2}$.

(b) When $\operatorname{gcd}\left(2, s_{1}+\lambda\right)=1$, the discriminant is a branch of semigroup $\left\langle 2, s_{1}+\lambda\right\rangle$.

Proof Suppose $\lambda=0$. The implicit equation of the normal form is $f(x, y)=y^{3}-x^{s_{1}}=0$. Then $f_{y}(x, y)=3 y^{2}$ whose Newton-Puiseux root is $y=0$, with multiplicity 2 . Hence, after (2.3) we have $D(u, v)=(v-f(u, 0))^{2}=\left(v+u^{s_{1}}\right)^{2}$, that is the discriminant is a double smooth branch.

Suppose now $\lambda \neq 0$. The normal forms are $x=t^{3}, y=t^{s_{1}}+t^{\lambda}$, with $\lambda$ as in (3.1). After (1.1) the implicit equation is $f(x, y)=y^{3}-3 x^{\frac{s_{1}+\lambda}{3}} y-\left(x^{s_{1}}+x^{\lambda}\right)$. Then $f_{y}(x, y)=$ $3 y^{2}-3 x^{\frac{s_{1}+\lambda}{3}}$, whose Newton-Puiseux roots are $\gamma_{i}(x)= \pm x^{\frac{s_{1}+\lambda}{6}}$ for $1 \leq i \leq 2$. Using (2.3) we have

$$
D(u, v)=\left(v+u^{s_{1}}-2 u^{\frac{s_{1}+\lambda}{2}}+u^{\lambda}\right)\left(v+u^{s_{1}}+2 u^{\frac{s_{1}+\lambda}{2}}+u^{\lambda}\right) .
$$




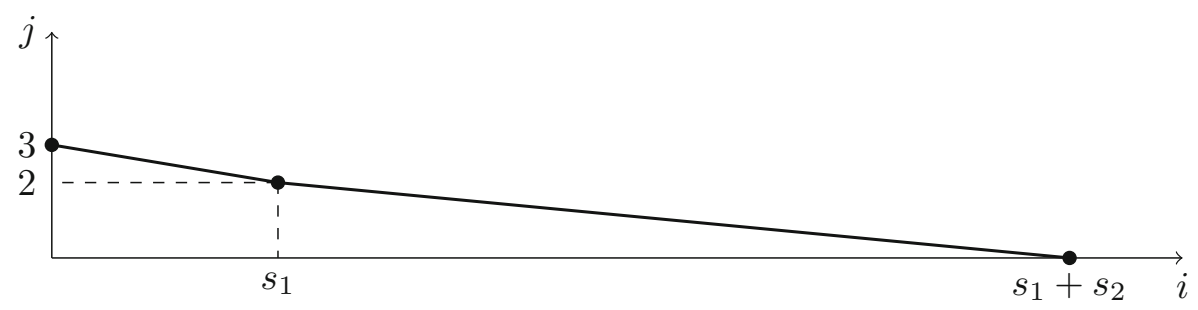

Fig. 1 Jacobian Newton polygon of a branch with semigroup $\left\langle 4, s_{1}, s_{2}\right\rangle$

Hence $\operatorname{Zer}(D)=\left\{\eta_{1}:=-u^{s_{1}}-2 u^{\frac{s_{1}+\lambda}{2}}-u^{\lambda}, \eta_{2}:=-u^{s_{1}}+2 u^{\frac{s_{1}+\lambda}{2}}-u^{\lambda}\right\}$ and $\operatorname{ord}\left(\eta_{1}-\right.$ $\left.\eta_{2}\right)=\frac{s_{1}+\lambda}{2}$. We distinguish two cases: if $\operatorname{gcd}\left(2, s_{1}+\lambda\right)=2$ then by (1.2) we conclude that $D(u, v)=0$ has two smooth branches of equations $D_{1}(u, v):=v+u^{s_{1}}-2 u^{\frac{s_{1}+\lambda}{2}}+u^{\lambda}$ and $D_{2}(u, v)=v+u^{s_{1}}+2 u^{\frac{s_{1}+\lambda}{2}}+u^{\lambda}$ such that, after the Halphen-Zeuthen formula, the intersection multiplicity is $i_{0}\left(D_{1}, D_{2}\right)=\frac{s_{1}+\lambda}{2}$. If $\operatorname{gcd}\left(2, s_{1}+\lambda\right)=1$ then the discriminant curve $D(u, v)=0$ is a singular branch of the semigroup $\left\langle 2, s_{1}+\lambda\right\rangle$.

The Newton polygon of $D(u, v)$ is elementary (it has only one compact edge) and the univariate polynomial associated with its compact edge is $(z+1)^{2}$. Hence the discriminant $D(u, v)=0$ is degenerate.

Corollary 3.2 If $C$ is a branch of multiplicity 2 or 3 and nonzero Zariski invariant, then the discriminant curve $D(u, v)=0$ has no multiple irreducible branches.

Corollary 3.2 does not hold for branches of multiplicity 4 as the proof of Proposition 3.5 shows.

\subsection{Discriminants of branches of multiplicity 4}

Let $C \equiv f(x, y)=0$ be a branch of multiplicity 4 . The branch $C$ may have genus 1 or 2 .

Proposition 3.3 Let $C \equiv f(x, y)=0$ be a branch of the semigroup $\left\langle 4, s_{1}, s_{2}\right\rangle$. Then the discriminant curve $D(u, v)=0$ is nondegenerate. Moreover, $D(u, v)=D_{1}(u, v) D_{2}(u, v)$, where $D_{1}$ is a smooth branch, $D_{2}$ is a singular branch of semigroup $\left\langle 2, s_{2}\right\rangle$ and the intersection multiplicity between the two branches is $i_{0}\left(D_{1}, D_{2}\right)=2 s_{1}$.

Proof For genus 2, and after the second part of [5, Corollary 4.4] we get that $D(u, v)=0$ is nondegenerate and we can determine its topological type from its Newton polygon (see [13, Proposition 4.7] and [6, Theorem 3.2]), which is the jacobian Newton polygon of $(x, f)$ (see Fig. 1).

Since $s_{2}$ is an odd natural number, $\operatorname{gcd}\left(s_{1}+s_{2}, 2\right)=1$. Moreover, after the properties of $S(f)$, we get $e_{0} s_{1}=4 s_{1}<2 s_{2}=e_{1} s_{2}$, hence $s_{2}>2 s_{1}$ and $D(u, v)=D_{1}(u, v) D_{2}(u, v)$, where $D_{1}(u, v)=0$ is a smooth curve admitting as parametrization $\left(t, t^{s_{1}}+\cdots\right)$ and $D_{2}(u, v)=0$ is a singular curve of genus 1 which semigroup of values is $\left\langle 2, s_{2}\right\rangle$. Finally, after the Halphen-Zeuthen formula, the intersection multiplicity between the two branches is $i_{0}\left(D_{1}, D_{2}\right)=2 s_{1}$.

Suppose now that the branch $C$ has genus 1 and semigroup of values equals $\left\langle 4, s_{1}\right\rangle$. By [8], the moduli space of branches of multiplicity 4 and genus 1 has five families of normal forms: 


$$
\mathrm{NF} 4 . i: \quad x=t^{4}, \quad y_{i}=\sigma_{i}(t), \quad 1 \leq i \leq 5,
$$

where $\lambda_{i}$ is the Zariski invariant of the $i$ th normal form family. More precisely, we have

(1) $\left(\lambda_{1}, \sigma_{1}(t)\right)=\left(0, t^{s_{1}}\right)$,

(2) If $2 \leq i \leq 4$ then $\lambda_{i}=2 s_{1}-4 j$ for $2 \leq j \leq\left[\frac{s_{1}}{4}\right]$ and

$$
\sigma_{2}(t)=t^{s_{1}}+t^{\lambda_{2}}+a_{k} t^{3 s_{1}-\left(4\left[\frac{s_{1}}{4}\right]+j+1-k\right)}+\cdots+a_{j-\left[\frac{s_{1}}{4}\right]-2} t^{3 s_{1}-4\left(\left[\frac{s_{1}}{4}\right]+3-k\right)},
$$

with $a_{k} \neq 0$, and $1 \leq k \leq\left[\frac{s_{1}}{4}\right]-j$.

$$
\begin{aligned}
& \sigma_{3}(t)=t^{s_{1}}+t^{\lambda_{3}}+\frac{3 s_{1}-4 j}{2 s_{1}} t^{3 s_{1}-8 j}+a_{\left[\frac{s_{1}}{4}\right]-j+2} t^{3 s_{1}-4(2 j-1)}+\cdots \\
& +a_{\left[\frac{s_{1}}{4}\right]} t^{3 s_{1}-(j+1)} \cdot \\
& \sigma_{4}(t)=t^{s_{1}}+t^{\lambda_{4}}+a_{\left[\frac{s_{1}}{4}\right]-j+1} t^{3 s_{1}-8 j}+a_{\left[\frac{s_{1}}{4}\right]-j+2} t^{3 s_{1}-4(2 j-1)}+\cdots \\
& +a_{\left[\frac{s_{1}}{4}\right]-1} t^{3 s_{1}-4(j+2)},
\end{aligned}
$$

where $a_{\left[\frac{s_{1}}{4}\right]-j+1} \neq \frac{3 s_{1}-4 j}{2 s_{1}}$.

(3) If $i=5$ then $\lambda_{5}=3 s_{1}-4 j$ for $2 \leq j \leq\left[\frac{s_{1}}{2}\right]$ and

$$
\begin{gathered}
\sigma_{5}(t)=t^{s_{1}}+t^{3 s_{1}-4 j}+a_{k} t^{2 s_{1}-4\left(j-\left[\frac{s_{1}}{4}\right]-k\right)}+a_{k+s} t^{2 s_{1}-4\left(j-\left[\frac{s_{1}}{4}\right] k-s\right)} \\
+\cdots
\end{gathered}
$$

After the Newton-Puiseux Theorem, the $i$ th normal form has the equation

$$
f_{i}(x, y)=\prod_{\omega^{4}=1}\left(y-\sigma_{i}\left(\omega x^{1 / 4}\right)\right),
$$

where $\omega$ is a 4 th primitive root of unity.

Hence, we obtain the implicit equation for each normal form family:

NF 4.1: $f_{1}(x, y)=y^{4}-x^{s_{1}}$.

NF 4.i: For $2 \leq i \leq 4$ we get

$$
f_{i}(x, y)=y^{4}+P_{i}(x) y^{2}+Q_{i}(x) y+x^{s_{1}} u(x),
$$

where $u(x) \in \mathbb{C}\{x\}$ is a unit (that is $u(0) \neq 0), \lambda_{i}=2 s_{1}-4 j$ for $2 \leq j \leq\left[\frac{s_{1}}{4}\right]$, $Q_{i}(x)=-4 x^{s_{1}-j}+\cdots$ and 


$$
P_{i}(x)= \begin{cases}-4 a_{k} x^{s_{1}-\left(\left[\frac{s_{1}}{4}\right]+j+1-k\right)}+\cdots & \text { for } i=2 \\ b x^{s_{1}-2 j}+\cdots & \text { for } i=3 \text { where } b=\frac{-8\left(s_{1}-j\right)}{s_{1}} . \\ \left(-2-4 a_{\left[\frac{s_{1}}{4}\right]-j+1}\right) x^{s_{1}-2 j}+\cdots & \text { for } i=4 \text { and }-2-4 a_{\left[\frac{s_{1}}{4}\right]-j+1} \neq 0 \\ 0 & \text { for } i=4, \quad-2-4 a_{\left[\frac{s_{1}}{4}\right]-j+1}=0 \\ & \text { and } a_{\left[\frac{s_{1}}{4}\right]-j+2}=\cdots=a_{\left[\frac{s_{1}}{4}\right]-1}=0 \\ -4 a_{\left[\frac{s_{1}}{4}\right]-j+k_{0}} x^{s_{1}-\left(2 j-\left(k_{0}-1\right)\right)}+\cdots & \text { for } i=4,-2-4 a_{\left[\frac{s_{1}}{4}\right]-j+1}=0 \\ & \text { and } a_{l} \neq 0 \text { for some } l=\left[\frac{s_{1}}{4}\right]-j+2, \ldots,\left[\frac{s_{1}}{4}\right]-1\end{cases}
$$

where $2 \leq k_{0} \leq j-1$ such that

$$
k:=\min \left\{l: a_{l} \neq 0,\left[\frac{s_{1}}{4}\right]-j+2 \leq l<\left[\frac{s_{1}}{4}\right]\right\}=\left[\frac{s_{1}}{4}\right]-j+k_{0} .
$$

Observe that

$$
\operatorname{ord} P_{i}(x) \geq \frac{2}{3}\left(s_{1}-j\right) .
$$

NF 4.5: $f_{5}(x, y)=y^{4}+P(x) y^{2}+Q(x) y+R(x)$, where

$$
\begin{aligned}
P(x)=- & 4 x^{s_{1}-j}-2 a_{k}^{2} x^{s_{1}-2\left(j-\left[\frac{s_{1}}{4}\right]-k\right)}-4 a_{k} a_{k+s} x^{s_{1}-2\left(j-s_{1}-\left[\frac{s_{1}}{4}\right]-k\right)+s} \\
& -2 a_{k+s}^{2} x^{s_{1}-2\left(j-\left[\frac{s_{1}}{4}\right]-k-s\right)}+\cdots \\
Q(x)=- & 4 a_{k} x^{s_{1}-j+\left[\frac{s_{1}}{4}\right]+k}-4 a_{k+s} x^{s_{1}-j-\left[\frac{s_{1}}{4}\right]+k+s} \\
& \left.-4 a_{k} x^{2\left(s_{1}-j\right)-\left(j-\left[\frac{s_{1}}{4}\right]-k\right)}-4 a_{k+s} x^{2\left(s_{1}-j\right)-\left(j-\left[\frac{s_{1}}{4}\right]-k-s\right)}+\cdots\right) \\
R(x)=- & x^{s_{1}}+2 x^{2\left(s_{1}-j\right)}-x^{3 s_{1}-4 j}+a_{k} x^{2 s_{1}-4\left(j-\left[\frac{s_{1}}{4}\right]-k\right)}+\cdots
\end{aligned}
$$

for $2 \leq j \leq\left[\frac{s_{1}}{2}\right]$.

Proposition 3.4 Let $C \equiv f(x, y)=0$ be a branch belonging to the family NF 4.i, for $1 \leq i \leq 4$. Then the discriminant curve $D(u, v)=0$ is degenerate and its topological type is determined by the semigroup $S(f)=\left\langle 4, s_{1}\right\rangle$ and the Zariski invariant $\lambda_{i}$ of $C$. Moreover,

(1) if $\lambda_{i}=0$ then the discriminant curve is the triple smooth branch $\left(v+u^{s_{1}}\right)^{3}=0$;

(2) if $\lambda_{i} \neq 0$ then $\lambda_{i}=2 s_{1}-4 j$ for $2 \leq j \leq\left[\frac{s_{1}}{4}\right]$ and

(a) when $\operatorname{gcd}\left(3,2 s_{1}+\lambda_{i}\right)=1$, the discriminant curve $D(u, v)=0$ is a branch with semigroup $\left\langle 3,2 s_{1}+\lambda_{i}\right\rangle$;

(b) when $\operatorname{gcd}\left(3,2 s_{1}+\lambda_{i}\right)=3$, the discriminant curve is the union of three smooth branches $D_{i}(u, v)=0,1 \leq i \leq 3$ with intersection number $i_{0}\left(D_{l}, D_{r}\right)=\frac{2 s_{1}+\lambda_{i}}{3}$ for $l \neq r$.

Proof Suppose $\lambda_{i}=0$. By (3.3) the implicit equation of the normal form is $f_{1}(x, y)=$ $y^{4}-x^{s_{1}}$. Then $\frac{\partial f_{1}}{\partial y}(x, y)=4 y^{3}$ whose Newton-Puiseux root is $y=0$, with multiplicity three. Hence, after (2.3) we have $D(u, v)=\left(v-f_{1}(u, 0)\right)^{3}=\left(v+u^{s_{1}}\right)^{3}$, that is the discriminant curve is a triple smooth branch.

Suppose now $\lambda_{i} \neq 0$. Then $\lambda_{i}=2 s_{1}-4 j$ for $2 \leq j \leq\left[\frac{s_{1}}{4}\right]$ and the normal form of $C$ is $x=t^{4}, \quad y=\sigma_{i}(t)$ for $2 \leq i \leq 4$.

From the implicit equations $f_{i}(x, y), 2 \leq i \leq 4$, given in (3.4) and from inequality (3.5) we get that the Newton polygon of $\frac{\partial f_{i}}{\partial y}(x, y)$ has only one compact edge whose vertices are 
$(0,3)$ and $\left(s_{1}-j, 0\right)$. All the parametrisations of $\frac{\partial f_{i}}{\partial y}(x, y)=0$, for $2 \leq i \leq 4$, have the same order and we can write them as $\gamma_{r}(u)=\varepsilon u^{\frac{s_{1}-j}{3}}+\cdots$, where $\varepsilon$ is a 3rd root of unity. From (2.3) and for a fixed $i \in\{2,3,4\}$, we have

$$
D(u, v)=\prod_{\frac{\partial f_{i}}{\partial y}\left(\gamma_{r}\right)=0}\left(v-f_{i}\left(u, \gamma_{r}(u)\right)\right),
$$

and considering the development of $f_{i}\left(u, \gamma_{r}(u)\right)$ we obtain

$$
D(u, v)=\prod_{\varepsilon^{3}=1}\left(v+u^{s_{1}}+3 \varepsilon u^{4\left(\frac{s_{1}-j}{3}\right)}+\cdots\right)=\prod_{\varepsilon^{3}=1}\left(v+u^{s_{1}}+3 \varepsilon u^{\left(\frac{2 s_{1}+\lambda_{i}}{3}\right)}+\cdots\right),
$$

so $\operatorname{Zer}(D)=\left\{\eta_{i}(\varepsilon):=-u^{s_{1}}-3 \varepsilon u^{\left(\frac{2 s_{1}+\lambda_{i}}{3}\right)}+\cdots\right\}_{\varepsilon^{3}=1}$ and $\operatorname{ord}\left(\eta_{l}-\eta_{r}\right)=\frac{2 s_{1}+\lambda_{i}}{3}$, for $1 \leq l \neq r \leq 3$. The topological type of $D(u, v)=0$ is determined by the semigroup of the branch $C \equiv f_{i}(x, y)=0$ and its Zariski analytical invariant $\lambda_{i}=2 s_{1}-4 j$ for $2 \leq j \leq\left[\frac{s_{1}}{4}\right]$. We distinguish two cases: if 3 and $2 s_{1}+\lambda_{i}$ are coprime then the discriminant $D(u, v)=0$ is a branch of the semigroup $\left\langle 3,2 s_{1}+\lambda_{i}\right\rangle$. Otherwise, by (1.2), we conclude that the discriminant curve is the union of three different smooth branches $D_{l}(u, v)=0$ with intersection multiplicity $i_{0}\left(D_{l}, D_{r}\right)=\frac{2 s_{1}+\lambda_{i}}{3}$ for $l \neq r$.

In all cases the Newton polygon of $D(u, v)$ is elementary with vertices $(0,3)$ and $\left(3 s_{1}, 0\right)$. The polynomial associated with its compact edge is $(z+1)^{3}$, so the discriminant curve $D(u, v)=0$ is degenerate.

Proposition 3.5 Let $C \equiv f(x, y)=0$ be a branch belonging to the family NF 4.5. Then the discriminant curve $D(u, v)=0$ is degenerate and its topological type is determined by the semigroup $S(f)=\left\langle 4, s_{1}\right\rangle$, the Zariski invariant $\lambda_{5}$ and at most two other analytical invariants of $C$.

Proof The implicit equation of $C$ has the form $f_{5}(x, y)=y^{4}+P(x) y^{2}+Q(x) y+R(x)$, where $P(x), Q(x), R(x)$ are as in (3.6), (3.7) and (3.8). Hence $\frac{\partial f_{5}}{\partial y}(x, y)=4 y^{3}+2 P(x) y+$ $Q(x)$.

We distinguish the following cases:

Case $A$. If $a_{i}=0$ in $\sigma_{5}(t)$ for all $i$, then we have that $\frac{\partial f_{5}}{\partial y}(x, y)=4 y^{3}-8 x^{s_{1}-j} y=$ $4 y\left(y^{2}-2 x^{s_{1}-j}\right)$ whose Newton-Puiseux roots are $\left\{0, \pm \sqrt{2} x^{\frac{s_{1}-j}{2}}\right\}$. Therefore $f_{5}(u, 0)=$ $R(u)=-u^{s_{1}}+2 u^{2\left(s_{1}-j\right)}-u^{3 s_{1}-4 j}$ and $f_{5}\left(u, \pm \sqrt{2} u^{\frac{s_{1}-j}{2}}\right)=-u^{s_{1}}-2 u^{2\left(s_{1}-j\right)}-u^{3 s_{1}-4 j}$. From (2.3) we conclude that the discriminant curve is the union of three smooth curves $D_{l}(u, v)=0$, where $D_{1}(u, v)=v+u^{s_{1}}-2 u^{2\left(s_{1}-j\right)}+u^{3 s_{1}-4 j}, D_{2}(u, v)=D_{3}(u, v)=$ $v+u^{s_{1}}+2 u^{2\left(s_{1}-j\right)}+u^{3 s_{1}-4 j}$, and $i_{0}\left(D_{1}, D_{l}\right)=2\left(s_{1}-j\right)$ for $l \in\{2,3\}$.

Case B. If $a_{k} \neq 0$ and $a_{k+l}=0$ in $\sigma_{5}(t)$ for $l>0$, then

$$
\begin{aligned}
\frac{\partial f_{5}}{\partial y}(x, y) & =4 y^{3}+2\left(-4 x^{s_{1}-j}-2 a_{k}^{2} x^{s_{1}-2\left(j-\left[\frac{s_{1}}{4}\right]-k\right)}+\cdots\right) y \\
& -4 a_{k}\left(x^{s_{1}-j+\left[\frac{s_{1}}{4}\right]+k}+x^{2\left(s_{1}-j\right)-\left(j-\left[\frac{s_{1}}{4}\right]-k\right)}+\cdots\right) .
\end{aligned}
$$

So the Newton polygon of $\frac{\partial f_{5}}{\partial y}(x, y)$ depends on the position of the point $M=\left(s_{i}-j, 1\right)$ with respect to the line passing by $E=(0,3)$ and $F=\left(s_{1}-j+\left[\frac{s_{1}}{4}\right]+k, 0\right)$. We have three subcases: 
B.1. If $\frac{2}{s_{1}-j}<\frac{1}{\left[\frac{s_{1}}{4}\right]+k}$ then $\mathcal{N}\left(\frac{\partial f_{5}}{\partial y}\right)$ has only one compact edge of vertices $E$ and $F$. Hence the order of the Newton-Puiseux roots $\left\{\gamma_{i}\right\}_{i=1}^{3}$ of $\frac{\partial f_{5}}{\partial y}(x, y)=0$ equals $\frac{s_{1}-j+\left[\frac{s_{1}}{4}\right]+k}{3}$. After (2.3) we obtain

$$
D(u, v)=\prod_{w_{i}^{3}=1}\left(v-3 a^{\frac{4}{3}} w_{i} u^{4\left(\frac{s_{1}-j+\left[\frac{s_{1}}{4}\right]+k}{3}\right)}+u^{s_{1}}+\cdots\right),
$$

for some nonzero complex number $a$ and where $w_{i}$ is a cubic root of unity. If $\operatorname{gcd}\left(3,\left(s_{1}-\right.\right.$ $\left.\left.j+\left[\frac{s_{1}}{4}\right]+k\right)\right)=1$ then the discriminant curve is irreducible with semigroup $\left\langle 3,4\left(s_{1}-j+\right.\right.$ $\left.\left.\left[\frac{s_{1}}{4}\right]+k\right)\right\rangle$. In the other case, we get $\operatorname{gcd}\left(3,\left(s_{1}-j+\left[\frac{s_{1}}{4}\right]+k\right)\right)=3$ and the discriminant curve is the union of three smooth curves $D_{l}(x, y)=0$ such that $i_{0}\left(D_{l}, D_{r}\right)=\frac{4\left(s_{1}-j+\left[\frac{s_{1}}{4}\right]+k\right)}{3}$.

B.2. If $\frac{2}{s_{1}-j}>\frac{1}{\left[\frac{s_{1}}{4}\right]+k}$ then $\mathcal{N}\left(\frac{\partial f_{5}}{\partial y}\right)$ has two compact edges of vertices $M, E$ and $F$. By (2.3) we get

$$
D(u, v)=\left(v+u^{s_{1}}-2 u^{2\left(s_{1}-j\right)}+\cdots\right)\left(v+u^{s_{1}}+2 u^{2\left(s_{1}-j\right)} \pm 4 \sqrt{2} a_{k} u^{\frac{3}{2}\left(s_{1}-j\right)+\left[\frac{s_{1}}{4}\right]+k}+\cdots\right) .
$$

If $s_{1}-j$ is odd then the discriminant curve is the union of a smooth branch $D_{1}(u, v)=0$ and a singular branch $D_{2}(x, y)=0$ with semigroup $\left\langle 2,3\left(s_{1}-j\right)+2\left(\left[\frac{s_{1}}{4}\right]+k\right)\right\rangle$. Moreover, $i_{0}\left(D_{1}, D_{2}\right)=4\left(s_{1}-j\right)$. Otherwise, if $s_{1}-j$ is even then the discriminant curve is the union of three smooth branches $D_{l}(x, y)=0$ such that $i_{0}\left(D_{1}, D_{l}\right)=2\left(s_{1}-j\right)$ for $2 \leq l \leq 3$ and $i_{0}\left(D_{2}, D_{3}\right)=\frac{3}{2}\left(s_{1}-j\right)+\left[\frac{s_{1}}{4}\right]+k$.

B.3. If $\frac{2}{s_{1}-j}=\frac{1}{\left[\frac{s_{1}}{4}\right]+k}$ then $\mathcal{N}\left(\frac{\partial f_{5}}{\partial y}\right)$ has only one compact edge of vertices $E$ and $F$ and $M$ is an interior point of this edge. The polynomial in one variable associated with the compact edge of $\mathcal{N}\left(\frac{\partial f_{5}}{\partial y}\right)$ is $p(z)=z^{3}-2 z-a_{k}$. After the $z$-discriminant of $p(z)$ we have that the roots of $p(z)$ are simple if and only if $a_{k} \neq \pm\left(\frac{4 \sqrt{6}}{9}\right)$. We will study both cases:

B.3.1 Suppose that $a_{k} \neq \pm\left(\frac{4 \sqrt{6}}{9}\right)$. Denote by $z_{i}$ the three different roots of the polynomial $p(z)$. For any $\gamma_{i}(x) \in \operatorname{Zer}\left(\frac{\partial f_{5}}{\partial y}\right)$ we have

$$
f\left(u, \gamma_{i}(u)\right)=-u^{s_{1}}+q\left(z_{i}\right) u^{2\left(s_{1}-j\right)}+\cdots \text {, where } q(z)=z^{4}-4 z^{2}-4 a_{k} z+2 .
$$

Since $z^{3}-2 z=a_{k}$, we get $q(z)=-3 z^{4}+4 z^{2}+2$ and if $z_{r} \neq z_{l}$ then $q\left(z_{r}\right) \neq q\left(z_{l}\right)$. Hence $D(u, v)=\prod_{i}\left(z+u_{1}^{s}+q\left(z_{i}\right) u^{2\left(s_{1}-j\right)}+\cdots\right)$ and the discriminant curve $D(u, v)=0$ is the union of three smooth branches $D_{l}(x, y)=0$ such that $i_{0}\left(D_{l}, D_{r}\right)=2\left(s_{1}-j\right)$.

B.3.2 Suppose that $a_{k}=\frac{4 \sqrt{6}}{9}$. The polynomial $p(z)$ has $z_{1}=2 \sqrt{\frac{2}{3}}$ as a simple root and $z_{2}=-\sqrt{\frac{2}{3}}$ as a double root. If $\gamma_{i} \in \operatorname{Zer}\left(\frac{\partial f_{5}}{\partial y}\right)$ corresponds to $z_{i}$ then

$$
\begin{aligned}
& f_{5}\left(u, \gamma_{1}(u)\right)=u^{s_{1}}+q\left(z_{1}\right) u^{2\left(s_{1}-j\right)}+\cdots, \\
& f_{5}\left(u, \gamma_{2}(u)\right)=u^{s_{1}}+q\left(z_{2}\right) u^{2\left(s_{1}-j\right)}+r_{2} u^{2\left(s_{1}-j\right)+\frac{s_{1}-2 j}{2}}+\cdots, \\
& f_{5}\left(u, \gamma_{3}(u)\right)=u^{s_{1}}+q\left(z_{2}\right) u^{2\left(s_{1}-j\right)}+r_{3} u^{2\left(s_{1}-j\right)+\frac{s_{1}-2 j}{2}}+\cdots,
\end{aligned}
$$

where $r_{2}, r_{3} \in \mathbb{C}$ are different. Observe that $q\left(z_{1}\right) \neq q\left(z_{2}\right)$. Hence, if $s_{1}-2 j$ is odd then the discriminant curve is the union of a smooth branch $D_{1}(u, v)=0$ and a singular branch $D_{2}(u, v)=0$ with semigroup $\left\langle 2,5 s_{1}-6 j\right\rangle$, where $i_{0}\left(D_{1}, D_{2}\right)=4\left(s_{1}-j\right)$. Otherwise, if $s_{1}-2 j$ is even then the discriminant curve is the union of three smooth branches $D_{l}(u, v)=0$ such that $i_{0}\left(D_{1}, D_{l}\right)=2\left(s_{1}-j\right)$, for $2 \leq l \leq 3$ and $i_{0}\left(D_{2}, D_{3}\right)=\frac{5 s_{1}-7 j}{2}$. 
B.3.3 Suppose that $a_{k}=-\frac{4 \sqrt{6}}{9}$. The polynomial $p(z)$ has $2 \sqrt{\frac{2}{3}}$ as a double root and $-\sqrt{\frac{2}{3}}$ as a simple root. After a similar procedure we conclude, in this case, that the topological type of the discriminant curve is as in B.3.2.

Case $C$ If $a_{k} \neq 0 \neq a_{k+s}$, then we have $\frac{\partial f_{5}}{\partial y}(x, y)=4 y^{3}+2 P(x) y+Q(x)$. The Newton polygon of $\frac{\partial f_{5}}{\partial y}(x, y)$ depends on the position of the point $M=\left(s_{i}-j, 1\right)$ with respect to the line passing through $E=(0,3)$ and $F=\left(s_{1}-j+\left[\frac{s_{1}}{4}\right]+k, 0\right)$. We have the following subcases:

C.1. If $\frac{2}{s_{1}-j}<\frac{1}{\left[\frac{s_{1}}{4}\right]+k}$ then the topological type of the discriminant curve $D(u, v)=0$ is as in In emphatic way.

C.2. If $\frac{2}{s_{1}-j}>\frac{1}{\left[\frac{s_{1}}{4}\right]+k}$ then the topological type of de discriminant curve is as in In emphatic way.

C.3. If $\frac{2}{s_{1}-j}=\frac{1}{\left[\frac{s_{1}}{4}\right]+k}$ then the Newton polygon of $\frac{\partial f_{5}}{\partial y}(x, y)$ has only one compact edge containing the points $E, F, M$, as in In emphatic way. The polynomial associated with this compact edge is $p(z)=z^{3}-2 z-a_{k}$ whose roots are simple if and only if $a_{k} \neq \pm\left(\frac{4 \sqrt{6}}{9}\right)$. Let us study the different cases:

C.3.1. If $a_{k} \neq \pm\left(\frac{4 \sqrt{6}}{9}\right)$ then the topological type of the discriminant curve $D(u, v)=0$ is as in In emphatic way.

C.3.2. Suppose that $a_{k}= \pm\left(\frac{4 \sqrt{6}}{9}\right)$. The polynomial $p(z)$ has $z_{1}=2 \sqrt{\frac{2}{3}}$ as a simple root and $z_{2}=z_{3}=-\sqrt{\frac{2}{3}}$ as a double root. If $\gamma_{i} \in \operatorname{Zer}\left(\frac{\partial f_{5}}{\partial y}\right)$ corresponds to $z_{i}$ then we can write

$$
\gamma_{i}=z_{i} u^{\left[\frac{s_{1}}{4}\right]+k}+\cdots
$$

Hence

$$
\begin{aligned}
& \delta_{1}:=f\left(u, \gamma_{1}(u)\right)=-u^{s_{1}}+q\left(z_{1}\right) u^{2\left(s_{1}-j\right)}+\cdots, \\
& \delta_{2}:=f\left(u, \gamma_{2}(u)\right)=-u^{s_{1}}+q\left(z_{2}\right) u^{2\left(s_{1}-j\right)}+\cdots, \\
& \delta_{3}:=f\left(u, \gamma_{3}(u)\right)=-u^{s_{1}}+q\left(z_{2}\right) u^{2\left(s_{1}-j\right)}+\cdots,
\end{aligned}
$$

where $\delta_{2}, \delta_{3} \in \operatorname{Zer} D(u, v)$ are different. Observe that $q\left(z_{1}\right) \neq q\left(z_{2}\right)$. So ord $\left(\delta_{1}-\delta_{l}\right)=$ $2\left(s_{1}-j\right)$ for $2 \leq l \leq 3$. Let us determine ord $\left(\delta_{2}-\delta_{3}\right)$. For that we need to find new terms in $\delta_{2}$ and $\delta_{3}$. We apply the Newton procedure for $\frac{\partial f_{5}}{\partial y}$ : let $y_{1}$ be a new variable. Substituting $(x, y):=\left(x, x^{\left[\frac{s_{1}}{4}\right]+k}\left(z_{2}+y_{1}\right)\right)$ in $\frac{\partial f_{5}}{\partial y}(x, y)$ we get

$$
\frac{\partial f_{5}}{\partial y}\left(x, x^{\left[\frac{s_{1}}{4}\right]+k}\left(z_{2}+y_{1}\right)\right)=x^{3\left(\left[\frac{s_{1}}{4}\right]+k\right)} g\left(x, y_{1}\right),
$$

with

$$
\begin{aligned}
g\left(x, y_{1}\right)=4 & {\left[\left(\frac{2}{3}\right) z_{2}+2 y_{1}+3 z_{2} y_{1}^{2}+y_{1}^{3}\right]-\left(4 a_{k}+4 a_{k+s} x^{s}+4 a_{k} x^{s_{1}-2 j}+\cdots\right) } \\
& -\left[8+4 a_{k}^{2} x^{s_{1}-2 j}+8 a_{k} a_{k+s} x^{s_{1}-2 j+s}+4 a_{k+s}^{2} x^{s_{1}-2 j+2 s}+\cdots\right] y_{1} \\
& -\left[8+4 a_{k}^{2} x^{s_{1}-2 j}+8 a_{k} a_{k+s} x^{s_{1}-2 j+s}+4 a_{k+s}^{2} x^{s_{1}-2 j+2 s}+\cdots\right] z_{2} \\
=4 & {\left[3 z_{2} y_{1}^{2}+y_{1}^{3}\right]-\left(4 a_{k+s} x^{s}+4 a_{k} x^{s_{1}-2 j}+\cdots\right) } \\
& -\left[4 a_{k}^{2} x^{s_{1}-2 j}+8 a_{k} a_{k+s} x^{s_{1}-2 j+s}+4 a_{k+s}^{2} x^{s_{1}-2 j+2 s}+\cdots\right] y_{1} \\
& -\left[4 a_{k}^{2} x^{s_{1}-2 j}+8 a_{k} a_{k+s} x^{s_{1}-2 j+s}+4 a_{k+s}^{2} x^{s_{1}-2 j+2 s}+\cdots\right] z_{2},
\end{aligned}
$$

where the last equality follows from $p\left(z_{2}\right)=0$. 
Hence, in the next step of the Newton procedure it is enough to consider the polynomial

$$
H\left(x, y_{1}\right)=12 z_{2} y_{1}^{2}-4 a_{k}^{2} x^{s_{1}-2 j} y_{1}+\left(-4 z_{2} a_{k}^{2}-4 a_{k}\right) x^{s_{1}-2 j}-4 a_{k+s} x^{s} .
$$

The topological type of the discriminant curve will depend on the relation between $s$ and $s_{1}-2 j$ :

C.3.2.1 Suppose that $s_{1}-2 j>s$. We obtain

$$
\begin{aligned}
& \gamma_{2}(u)=-\frac{\sqrt{6}}{3} u^{\frac{s_{1}-j}{2}}+\frac{\sqrt{-a_{k+s}}}{\sqrt[4]{6}} u^{\frac{s_{1}-j}{2}+\frac{s}{2}}+\cdots, \\
& \gamma_{3}(u)=-\frac{\sqrt{6}}{3} u^{\frac{s_{1}-j}{2}}-\frac{\sqrt{-a_{k+s}}}{\sqrt[4]{6}} u^{\frac{s_{1}-j}{2}+\frac{s}{2}}+\cdots .
\end{aligned}
$$

Hence

$$
\begin{aligned}
f_{5}\left(u, \gamma_{2}(u)\right)= & -u_{1}^{s}+q\left(z_{2}\right) u^{2\left(s_{1}-j\right)}+p\left(z_{2}\right) u^{2\left(s_{1}-j\right)+\frac{s}{2}}+l\left(a_{k+s}\right) u^{3 s_{1}-4 j} \\
& +\frac{8}{3} c a_{k+s} u^{2\left(s_{1}-j\right)+\frac{3}{2} s}+\cdots
\end{aligned}
$$

and

$$
\begin{aligned}
f_{5}\left(u, \gamma_{3}(u)\right)= & -u_{1}^{s}+q\left(z_{2}\right) u^{2\left(s_{1}-j\right)}-p\left(z_{2}\right) u^{2\left(s_{1}-j\right)+\frac{s}{2}}+l\left(a_{k+s}\right) u^{3 s_{1}-4 j} \\
& -\frac{8}{3} c a_{k+s} u^{2\left(s_{1}-j\right)+\frac{3}{2} s}+\cdots
\end{aligned}
$$

where $c:=\frac{\sqrt{-a_{k+s}}}{\sqrt[4]{6}}$, and $l(z)=-4 z_{2} z-1$. As a consequence, when $s$ is odd then the discriminant curve $D(u, v)=0$ is the union of a smooth branch $D_{1}(u, v)=0$ and a singular branch with semigroup $\left\langle 2,4\left(s_{1}-j\right)+3 s\right\rangle$, with $i_{0}\left(D_{1}, D_{2}\right)=4\left(s_{1}-j\right)$. Otherwise, if $s$ is even then the discriminant curve $D(u, v)=0$ is the union of three smooth braches $D_{l}(u, v)=0$ such that $i_{0}\left(D_{1}, D_{l}\right)=2\left(s_{1}-j\right)$ for $2 \leq l \leq 3$, and... and $i_{0}\left(D_{2}, D_{3}\right)=2\left(s_{1}-j\right)+\frac{3}{2} s$.

C.3.2.2 Suppose that $s_{1}-2 j<s$. After (3.11) and for the next step of the Newton procedure we only need the polynomial

$$
\bar{H}(Z)=12 z_{2} Z^{2}-4\left(z_{2} a_{k}^{2}+a_{k}\right),
$$

whose roots are $\pm \frac{\sqrt{8}}{9}$. Let $d=\frac{\sqrt{8}}{9}$. We obtain

$$
\begin{aligned}
& \gamma_{2}(u)=z_{2} u^{\left[\frac{s_{1}}{4}\right]+k}+d u^{\left[\frac{s_{1}}{4}\right]+k+\frac{s_{1}-2 j}{2}}+\cdots, \\
& \gamma_{3}(u)=z_{2} u^{\left[\frac{s_{1}}{4}\right]+k}-d u^{\left[\frac{s_{1}}{4}\right]+k+\frac{s_{1}-2 j}{2}}+\cdots .
\end{aligned}
$$

Hence

$$
\begin{aligned}
f_{5}\left(u, \gamma_{2}(u)\right)= & -u_{1}^{s}+q\left(z_{2}\right) u^{2\left(s_{1}-j\right)}+p\left(z_{2}\right) u^{2\left(s_{1}-j\right)+\frac{s}{2}}+l\left(a_{k}\right) u^{3 s_{1}-4 j} \\
& +\frac{80}{81} z_{2} d u^{\frac{7 s_{1}-10 j}{2}}+\cdots,
\end{aligned}
$$

and

$$
\begin{aligned}
f_{5}\left(u, \gamma_{3}(u)\right)= & -u_{1}^{s}+q\left(z_{2}\right) u^{2\left(s_{1}-j\right)}-p\left(z_{2}\right) u^{2\left(s_{1}-j\right)+\frac{s}{2}}+l\left(a_{k}\right) u^{3 s_{1}-4 j} \\
& -\frac{80}{81} z_{2} d u^{\frac{7 s_{1}-10 j}{2}}+\cdots
\end{aligned}
$$


Since 4 and $s_{1}$ are coprime then $7 s_{1}-10$ is odd and the discriminant curve $D(u, v)=$ 0 is the union of a smooth branch $D_{1}(u, v)=0$ and a singular branch with semigroup $\left\langle 2,7 s_{1}-10 j\right\rangle$, where $i_{0}\left(D_{1}, D_{2}\right)=4\left(s_{1}-j\right)$.

C.3.2.3 Suppose that $s_{1}-2 j=s$. After (3.11), in order to obtain the next term in the power series $\gamma_{i}$, it is enough to consider the polynomial

$$
\bar{H}(Z)=12 z_{2} Z^{2}-4\left(z_{2} a_{k}^{2}+a_{k+s}+a_{k}\right) .
$$

The topological type of the discriminant will depend on the value of $a_{k+s}$ :

C.3.2.3.1 For $a_{k+s} \neq-4 \frac{\sqrt{6}}{81}$, we get

$$
\bar{H}(Z)=12 z_{2} Z^{2}-4\left(a_{k+s}+4 \frac{\sqrt{6}}{81}\right),
$$

whose roots are $\pm b:= \pm\left(\frac{\sqrt{-d_{k+s}}}{\sqrt[4]{6}}\right)$, where $d_{k+s}=a_{k+s}+4 \frac{\sqrt{6}}{81}$. Hence

$$
\begin{aligned}
& \gamma_{2}(u)=z_{2} u^{\left[\frac{s_{1}}{4}\right]+k}+b u^{\left[\frac{s_{1}}{4}\right]+k+\frac{s}{2}}+\cdots, \\
& \gamma_{3}(u)=z_{2} u^{\left[\frac{s_{1}}{4}\right]+k}-b u^{\left[\frac{s_{1}}{4}\right]+k+\frac{s}{2}}+\cdots .
\end{aligned}
$$

We conclude that

$$
f_{5}\left(u, \gamma_{2}(u)\right)=-u_{1}^{s}+q\left(z_{2}\right) u^{2\left(s_{1}-j\right)}+l\left(a_{k+s}\right) u^{3 s_{1}-4 j}+\frac{80}{81} z_{2} u^{2 s_{1}-j+\frac{3}{2} s}+\cdots,
$$

and

$$
f_{5}\left(u, \gamma_{3}(u)\right)=-u_{1}^{s}+q\left(z_{2}\right) u^{2\left(s_{1}-j\right)}+l\left(a_{k+s}\right) u^{3 s_{1}-4 j}-\frac{80}{81} z_{2} u^{2 s_{1}-j+\frac{3}{2} s}+\cdots .
$$

So, if $s$ is odd then $D(u, v)=0$ is the union of a smooth branch $D_{1}(u, v)=0$ and a singular branch of semigroup $\left\langle 2,7 s_{1}-10 j\right\rangle$, with $i_{0}\left(D_{1}, D_{2}\right)=4\left(s_{1}-j\right)$. In the other case, if $s$ is even then $D(u, v)=0$ is the union of three smooth branches $D_{l}(u, v)=0$ such that $i_{0}\left(D_{1}, D_{l}\right)=2\left(s_{1}-j\right)$ for $2 \leq l \leq 3$, and $\ldots$, and $i_{0}\left(D_{2}, D_{3}\right)=\frac{7 s_{1}-10 j}{2}$.

C.3.2.3.2 For $a_{k+s}=-4 \frac{\sqrt{6}}{81}$, after (3.10), in order to obtain the next term in the power series $\gamma_{i}$, it is enough to consider the polynomial

$$
\bar{H}(Z)=12 z_{2} Z^{2}-4 a_{k}^{2} Z-4 a_{k+s}\left(1+2 a_{k} z_{2}\right),
$$

whose roots $t_{1}, t_{2}$ are simple. Hence

$$
\gamma_{2}(u)=z_{2} u^{\left[\frac{s_{1}}{4}+k\right]+k}+t_{1} u^{\left[\frac{s_{1}}{4}+k\right]+k+s}+\cdots,
$$

and

$$
\gamma_{3}(u)=z_{2} u^{\left[\frac{s_{1}}{4}+k\right]+k}-t_{2} u^{\left[\frac{s_{1}}{4}+k\right]+k+s}+\cdots .
$$

We conclude that

$$
f_{5}\left(u, \gamma_{2}(u)\right)=-u_{1}^{s}+q\left(z_{2}\right) u^{2\left(s_{1}-j\right)}-u^{2\left(s_{1}-j\right)+s}+h\left(t_{1}\right) u^{2\left(s_{1}-j\right)+s 2}+\cdots,
$$

and

$$
f_{5}\left(u, \gamma_{3}(u)\right)=-u_{1}^{s}+q\left(z_{2}\right) u^{2\left(s_{1}-j\right)}-u^{2\left(s_{1}-j\right)+s}+h\left(t_{2}\right) u^{2\left(s_{1}-j\right)+s 2}+\cdots,
$$

where $h(z)=-4 a_{k+s} z+a_{k}$. The discriminant curve $D(u, v)=0$ is the union of three smooth branches $D_{l}(u, v)=0$ such that $i_{0}\left(D_{1}, D_{l}\right)=2\left(s_{1}-j\right)$ for $2 \leq l \leq 3$ and... and $i_{0}\left(D_{2}, D_{3}\right)=4 s_{1}-6 j$. 
From the above computations we obtain

Theorem 3.6 Let $f(x, y)=0$ be a plane branch of multiplicity $n$. Then the following hold.

(1) If $n=2$ then the discriminant curve $D(u, v)=0$ is nondegenerate.

(2) If $n=3$ then the discriminant curve $D(u, v)=0$ is degenerate and its topological type depends on the semigroup $S(f)$ and the Zariski invariant (when it is not zero).

(3) If $n=4$ then the discriminant curve $D(u, v)=0$ is degenerate and its topological type depends on the semigroup $S(f)$, the Zariski invariant (when it is not zero) and at most two other analytical invariants.

Remark 3.7 In [8], Hefez and Hernandes give explicit normal forms for branches of multiplicity 2, 3 or 4 . Thanks to that, the description of the topological type of the discriminant curve of such a branch was possible. The normal forms for branches of multiplicity greater than 4 are not completely determined.

\section{Discriminant of branches $C$ with $\mu(C)-\tau(C) \leq 2$}

Let $C: f(x, y)=0$ be a plane branch. Put $r(C):=\mu(C)-\tau(C)$, where $\mu(C)$ and $\tau(C)$ are the Milnor number and the Tjurina number of $C$, respectively. Observe that $r(C)$ is a nonnegative integer. In zero characteristic, from [15, Theorem 4] we get $r(C)=0$ if and only if $C$ is analytically equivalent to the curve $y^{s_{0}}-x^{s_{1}}=0$ for two coprime integers $s_{0}$ and $s_{1}$ greater than 1. Later, in [1], the authors describe all plane branches defined over an algebraically closed field of characteristic zero, modulo analytic equivalence, having the property that the difference between their Milnor and Tjurina numbers is 1 or 2 . In particular the authors determined the normal forms of the branches of this family, which show us that the Zariski invariant of these branches are determined by the two first generators of their semigroup. By [1, Corollary 5] we know that if $r(C) \neq 0$ then $r(C) \geq 2^{g-1}$. Hence, if $r(C)=1$ then $g=1$ and if $r(C)=2$ then $g \leq 2$. In this section we will describe the topological type of the discriminant curve $D(u, v)=0$ of branches $C$ with $\mu(C)-\tau(C) \leq 2$.

Theorem 4.1 Let $C: f(x, y)=0$ be a plane branch with $r(C):=\mu(C)-\tau(C) \leq 2$. Then the discriminant curve $D(u, v)=0$ is degenerate and its topological type is given by the semigroup $S(f)$.

Proof Suppose first that $r(C)=1$. By [1, Corollary 8] the branch $C$ is analytically equivalent to the curve defined by the equation $f(x, y)=y^{s_{0}}-x^{s_{1}}+x^{s_{1}-2} y^{s_{0}-2}$, where $2 \leq s_{0}<s_{1}$ are coprime integers. Hence $f_{y}(x, y)=s_{0} y^{s_{0}-1}+\left(s_{0}-2\right) x^{s_{1}-2} y^{s_{0}-3}=y^{s_{0}-3}\left(s_{0} y^{2}+\right.$ $\left(s_{0}-2\right) x^{s_{1}-2}$ ), whose Newton-Puiseux roots are $\alpha_{1}=0$ (with multiplicity $s_{0}-3$ ), $\alpha_{2}=$ $\sqrt{\frac{2-s_{0}}{s_{0}}} x^{\frac{s_{1}-2}{2}}$ and $\alpha_{3}=-\sqrt{\frac{2-s_{0}}{s_{0}}} x^{\frac{s_{1}-2}{2}}$. After (2.3) we obtain that the Newton-Puiseux roots of the discriminant curve are

(1) $\delta_{1}=u^{s_{1}}$ with multiplicity $s_{0}-3$,

(2) $\delta_{2}=-u^{s_{1}}+\left(\left(\sqrt{\frac{2-s_{0}}{s_{0}}}\right)^{s_{0}}+\left(\sqrt{\frac{2-s_{0}}{s_{0}}}\right)^{s_{0}-2}\right) u^{\frac{\left(s_{1}-2\right) s_{0}}{2}}$,

(3) $\delta_{3}=-u^{s_{1}}-\left(\left(\sqrt{\frac{2-s_{0}}{s_{0}}}\right)^{s_{0}}+\left(\sqrt{\frac{2-s_{0}}{s_{0}}}\right)^{s_{0}-2}\right) u^{\frac{\left(s_{1}-2\right) s_{0}}{2}}$.

Hence, if $s_{1}$ and $s_{0}$ are odd then the discriminant curve is given by $D(u, v)=$ $D_{1}(u, v)^{s_{0}-3} D_{2}(u, v)$, where $D_{1}(u, v)=\left(v-u^{s_{1}}\right)$ and $D_{2}(u, v)$ is a branch of semigroup $\left\langle 2,\left(s_{1}-2\right) s_{0}\right\rangle$ and the intersection multiplicity is $i_{0}\left(D_{1}, D_{2}\right)=\min \left\{s_{1},\left(s_{1}-2\right) n\right\}$. 
Otherwise $D(u, v)$ is the product of $D_{1}(u, v)^{s_{0}-3}$ and two smooth branches $D_{2}(u, v)$ and $D_{3}(u, v)$, where $i_{0}\left(D_{1}, D_{k}\right)=\min \left\{s_{1}, \frac{\left(s_{1}-2\right) s_{0}}{2}\right\}$ for $2 \leq k \leq 3$ and $i_{0}\left(D_{2}, D_{3}\right)=\frac{\left(s_{1}-2\right) s_{0}}{2}$.

Suppose now that $r(C)=2$. In this case we get $g \leq 2$. If $g=2$ then by [1, Corollary 13] the multiplicity of $C$ is 4 and we study this case in Proposition 3.3.

For $g=1$, after [1, Theorem 17, Corollary 18], we have two normal forms:

(A) $f(x, y)=y^{s_{0}}-x^{s_{1}}+x^{s_{1}-3} y^{s_{0}-2}$, with $2<s_{0}<s_{1}$.

(B) $f(x, y)=y^{s_{0}}-x^{s_{1}}+x^{s_{1}-2} y^{s_{0}-3}+\left(\sum_{k \geq 2}^{2+\left[\frac{s_{1}}{s_{0}}\right]} a_{k} x^{s_{1}-k}\right) y^{s_{0}-2}$, with $4 \leq s_{0}<s_{1}$, $\frac{2 s_{0}}{s_{0}-3}<s_{1}$ and $a_{k} \in \mathbb{C}$.

In the case (A) we get $f_{y}(x, y)=y^{s_{0}-3}\left(s_{0} y^{2}+\left(s_{0}-2\right) x^{s_{1}-3}\right)$, whose Newton-Puiseux roots are $\alpha_{1}=0$ (with multiplicity $s_{0}-3$ ), $\alpha_{2}=\sqrt{\frac{2-s_{0}}{s_{0}}} x^{\frac{s_{1}-3}{2}}$ and $\alpha_{3}=-\sqrt{\frac{2-s_{0}}{s_{0}}} x^{\frac{s_{1}-3}{2}}$. After (2.3) we obtain that the Newton-Puiseux roots of the discriminant curve are

(1) $\delta_{1}=u^{s_{1}}$ with multiplicity $s_{0}-3$,

(2) $\delta_{2}=-u^{s_{1}}+\left(\left(\sqrt{\frac{2-s_{0}}{s_{0}}}\right)^{s_{0}}+\left(\sqrt{\frac{2-s_{0}}{s_{0}}}\right)^{s_{0}-2}\right) u^{\frac{\left(s_{1}-3\right) s_{0}}{2}}$,

(3) $\delta_{3}=-u^{s_{1}}-\left(\left(\sqrt{\frac{2-s_{0}}{s_{0}}}\right)^{s_{0}}+\left(\sqrt{\frac{2-s_{0}}{s_{0}}}\right)^{s_{0}-2}\right) u^{\frac{\left(s_{1}-3\right) s_{0}}{2}}$.

Hence, if $s_{0}$ is odd and $s_{1}$ is even then the discriminant curve is given by $D(u, v)=$ $D_{1}(u, v)^{s_{0}-3} D_{2}(u, v)$, where $D_{1}(u, v)=\left(v-u^{s_{1}}\right)$ and $D_{2}(u, v)$ is a branch of the semigroup $\left\langle 2,\left(s_{1}-3\right) s_{0}\right\rangle$ and the intersection multiplicity is $i_{0}\left(D_{1}, D_{2}\right)=\min \left\{s_{1},\left(s_{1}-3\right) s_{0}\right\}$. Otherwise $D(u, v)$ is the product of $D_{1}(u, v)^{s_{0}-3}$ and two smooth branches $D_{2}(u, v)$ and $D_{3}(u, v)$, where $i_{0}\left(D_{1}, D_{k}\right)=\min \left\{s_{1}, \frac{\left(s_{1}-3\right) s_{0}}{2}\right\}$ for $2 \leq k \leq 3$ and $i_{0}\left(D_{2}, D_{3}\right)=\frac{\left(s_{1}-3\right) s_{0}}{2}$.

In the case (B) we have

$$
f_{y}(x, y)=y^{s_{0}-4}\left(s_{0} y^{3}+\left(s_{0}-3\right) x^{s_{1}-2}+\left(s_{0}-2\right)\left(\sum_{k \geq 2}^{2+\left[\frac{s_{1}}{s_{0}}\right]} a_{k} x^{s_{1}-k}\right) y\right),
$$

hence its Newton polygon coincides with the Newton polygon determined by $(0,3),\left(s_{1}-2,0\right)$ and $\left(s_{1}-2-\left[\frac{s_{1}}{s_{0}}\right], 1\right)$. But, after the inequality $\frac{2 s_{0}}{s_{0}-3}<s_{1}$, we get that this Newton polygon has only two points which are its vertices: $(0,3)$ and $\left(s_{1}-2,0\right)$. The Newton-Puiseux roots of $f_{y}(x, y)=0$ are $\alpha_{1}=0$ (with multiplicity $s_{0}-4$ ) and $\alpha_{i}=\xi_{i} \sqrt[3]{\frac{s_{0}-3}{s_{0}}} u^{\frac{s_{0}-2}{3}}+\cdots$, where $\xi_{i}$ is a cubic root of unity, $1 \leq i \leq 3$. Then, after (2.3) the Newton-Puiseux roots of the discriminant curve are $\delta_{1}=u^{s_{1}}$ with multiplicity $s_{0}-4$ and

$$
\begin{aligned}
\delta_{i}= & -u^{s_{1}}+\left[\left(\xi_{i} \sqrt[3]{\frac{s_{0}-3}{s_{0}}}\right)^{s_{0}}+\left(\xi_{i} \sqrt[3]{\frac{s_{0}-3}{s_{0}}}\right)^{s_{0}-3}\right] u^{\frac{\left(s_{1}-2\right) s_{0}}{3}} \\
& +\left(\xi_{i} \sqrt[3]{\frac{s_{0}-3}{s_{0}}}\right)^{s_{0}-2} a_{2+\left[\frac{s_{1}}{s_{0}}\right]} u^{\frac{\left(s_{1}-2\right)\left(s_{0}-2\right)}{3}+\left(s_{1}-2\right)-\left[\frac{s_{1}}{s_{0}}\right]}+\cdots,
\end{aligned}
$$

for $1 \leq i \leq 3$. 
If $s_{1}-2$ (respectively $s_{0}$ ) and 3 are coprime then the discriminant curve is given by $D(u, v)=D_{1}(u, v)^{s_{0}-4} D_{2}(u, v)$, where $D_{1}(u, v)=\left(v-u^{s_{1}}\right)$ and $D_{2}(u, v)$ is a branch of the semigroup $\left\langle 3,\left(s_{1}-2\right) s_{0}\right\rangle$ and, after the Halphen-Zeuthen formula, the intersection multiplicity is $i_{0}\left(D_{1}, D_{2}\right)=3 s_{1}$. Otherwise $D(u, v)$ is the product of $D_{1}(u, v)^{s_{0}-4}$ and three smooth branches $D_{k}(u, v), 2 \leq k \leq 4$, where $i_{0}\left(D_{1}, D_{k}\right)=s_{1}$ for $2 \leq k \leq 4$ and $i_{0}\left(D_{l}, D_{r}\right)=\frac{\left(s_{1}-2\right) s_{0}}{3}$, for $2 \leq l \neq r \leq 4$.

Remark 4.2 Observe that in Theorem 4.1 Case (B) with $s_{0}=4$ coincides with case $\sigma_{3}$ in (3.2) for $j=2$. Hence this case was studied in Proposition 3.4.

\section{Tables}

In this section we collect, in some tables, the topological type of the discriminants for branches studied in this paper (See Tables 1, 2, 3, 4, 5, 6, 7, 8, 9, 10, 11). 
Table 1 Discriminants of branches of semigroup $\left\langle 2, s_{1}\right\rangle$

Multiplicity 2

Normal form Discriminant $D(u, v)$

$\lambda=0$

$v+u^{s_{1}}$

Table 2 Discriminants of branches of semigroup $\left\langle 3, s_{1}\right\rangle$

\begin{tabular}{ll}
\hline Multiplicity 3 & \\
\hline Normal form & Discriminant $D(u, v)$ \\
\hline$\lambda=0$ & $\left(v+u^{s_{1}}\right)^{2}$ \\
$\lambda \neq 0$ & $D_{1} D_{2}, \quad m\left(D_{i}\right)=1, i_{0}\left(D_{1}, D_{2}\right)=\frac{s_{1}+\lambda}{2} i f \operatorname{gcd}\left(2, s_{1}+\lambda\right)=2$ \\
& $D_{1}, \quad S\left(D_{1}\right)=\left\langle 2, s_{1}+\lambda\right\rangle$ if $\operatorname{gcd}\left(2, s_{1}+\lambda\right)=1$
\end{tabular}

Table 3 Discriminants of branches of semigroup $\left\langle 4,6, s_{2}\right\rangle$

Multiplicity 4 and $g=2$

\begin{tabular}{ll}
\hline Normal form & Discriminant $D(u, v)$ \\
\hline$\lambda=s_{2}-6$ & $D_{1} D_{2}, \quad m\left(D_{1}\right)=1, \quad S\left(D_{2}\right)=\left\langle 2, s_{2}\right\rangle, \quad i_{0}\left(D_{1}, D_{2}\right)=2 s_{1}$
\end{tabular}

Table 4 Discriminants of branches of semigroup $\left\langle 4, s_{1}\right\rangle$

Multiplicity 4 and $g=1$

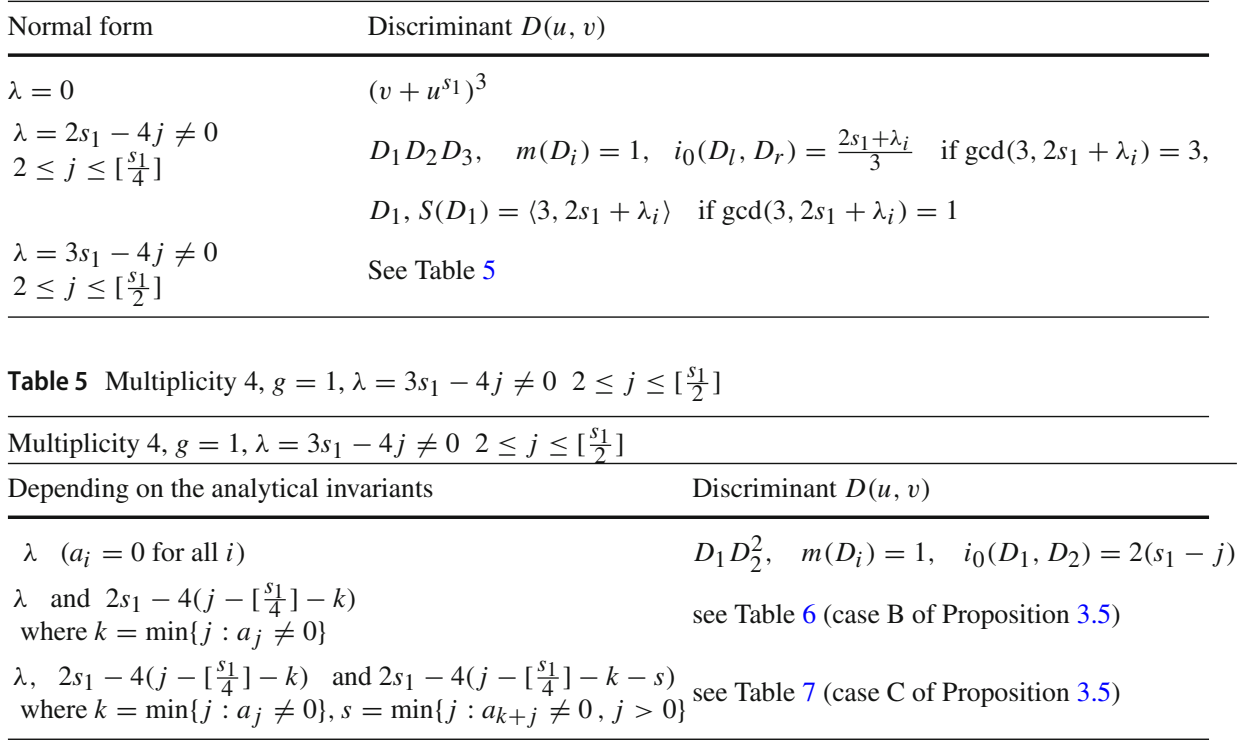




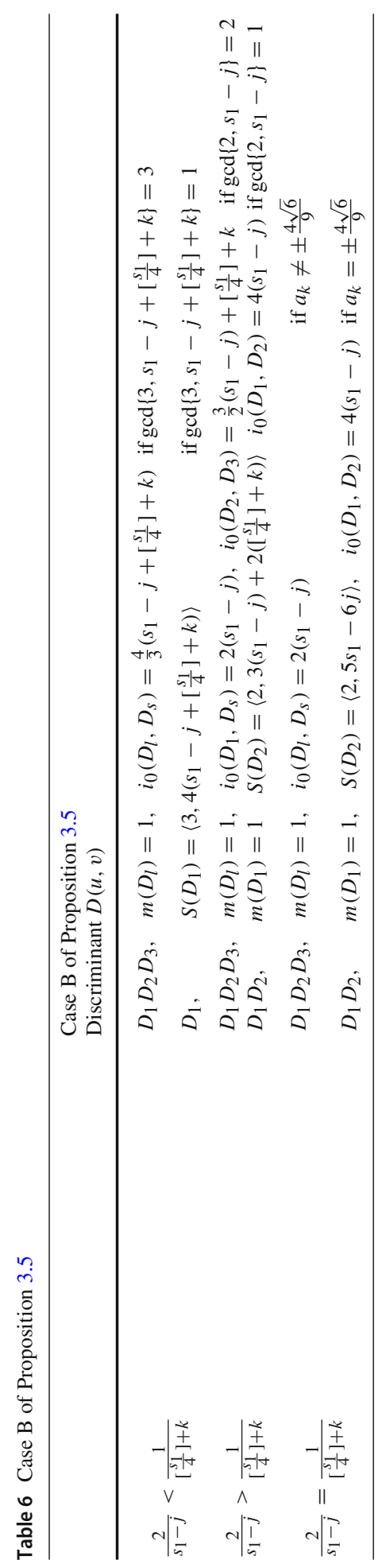




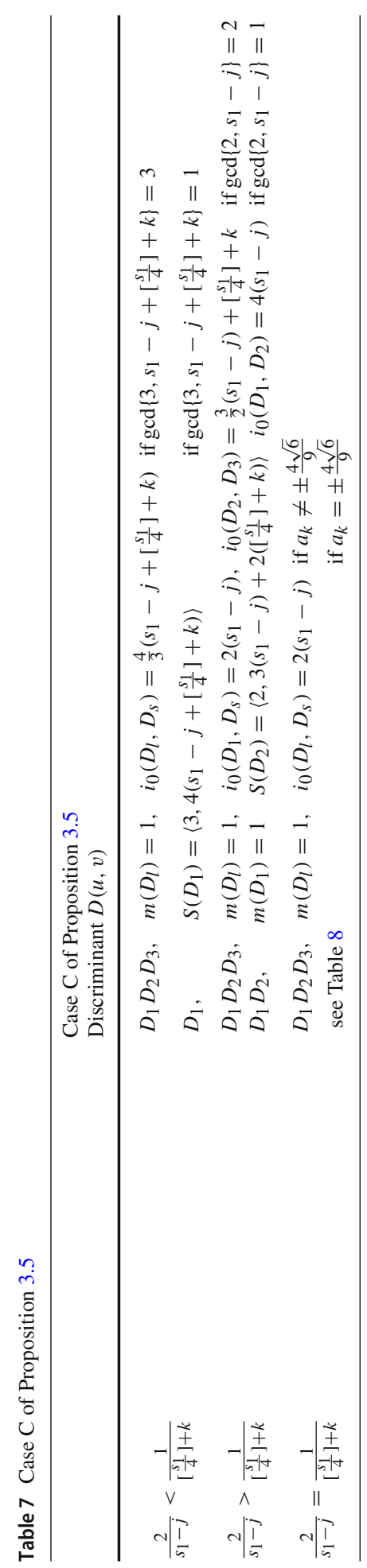




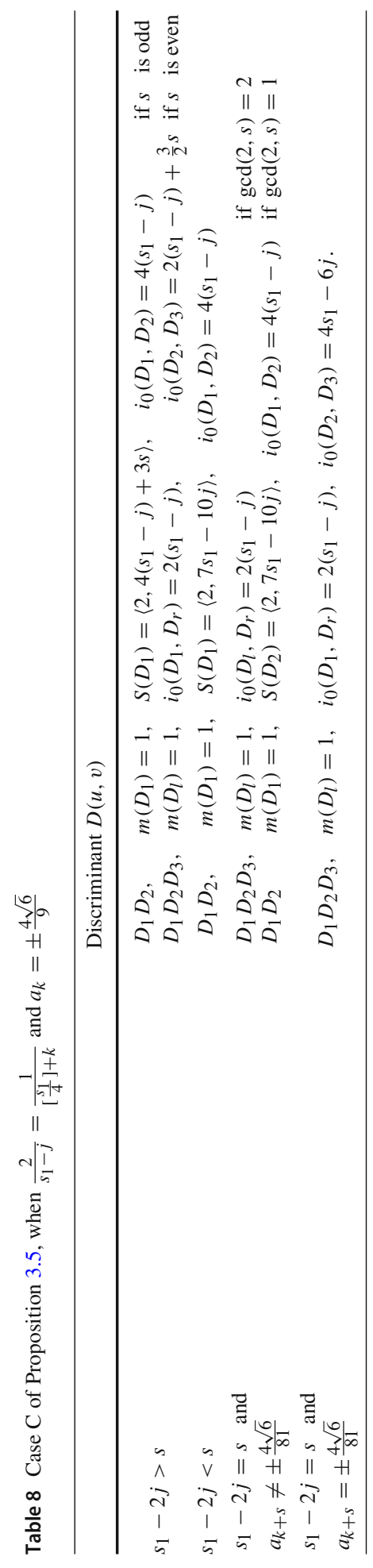




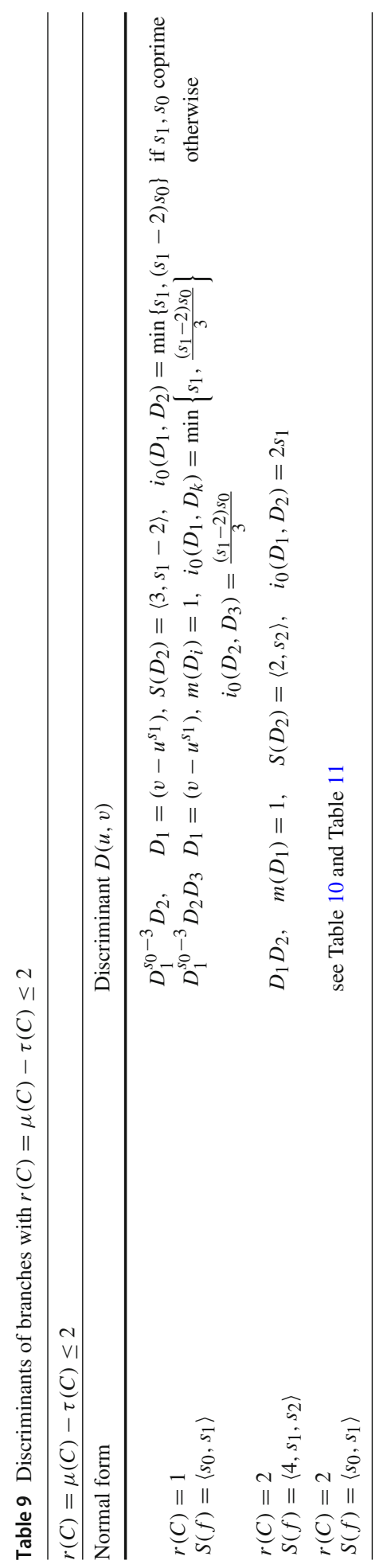




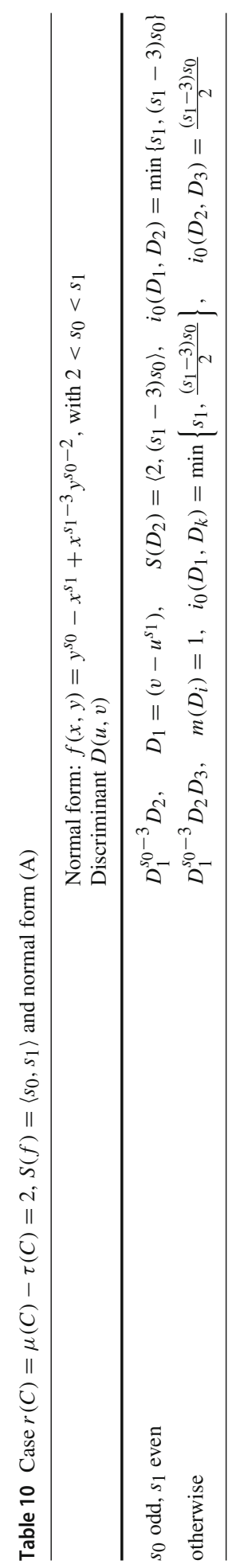




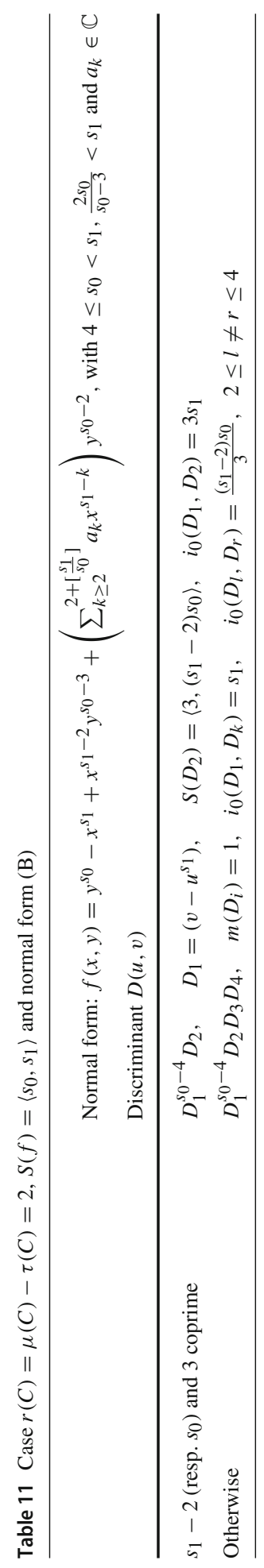


Funding Open Access funding provided thanks to the CRUE-CSIC agreement with Springer Nature.

Open Access This article is licensed under a Creative Commons Attribution 4.0 International License, which permits use, sharing, adaptation, distribution and reproduction in any medium or format, as long as you give appropriate credit to the original author(s) and the source, provide a link to the Creative Commons licence, and indicate if changes were made. The images or other third party material in this article are included in the article's Creative Commons licence, unless indicated otherwise in a credit line to the material. If material is not included in the article's Creative Commons licence and your intended use is not permitted by statutory regulation or exceeds the permitted use, you will need to obtain permission directly from the copyright holder. To view a copy of this licence, visit http://creativecommons.org/licenses/by/4.0/.

\section{References}

1. V. Bayer, A. Hefez. Algebroid plane curves whose Milnor and Tjurina numbers differ by one or two. Bol. Soc. Brasil. Mat. (N.S.) 32(1), 63-81 (2001)

2. E. Casas-Alvero, Local geometry of planar analytic morphisms. Asian J. Math. 11(3), 373-426 (2007)

3. A. Chenciner. Courbes algébriques planes. Publications Mathématiques de l'Université Paris VII, 1978

4. E. R. García Barroso, J. Gwoździewicz, A discriminant criterion of irreducibility. Kodai Math. J. 35(2), 403-414 (2012)

5. E.R. García Barroso, J. Gwoździewicz, A. Lenarcik, Non-degeneracy of the discriminant. Acta Math. Hungar. 147(1), 220-246. https://doi.org/10.1007/s10474-015-0515-8 (2015)

6. E.R. García Barroso, A. Lenarcik, A. Płoski, Characterization of non-degenerate plane curve singularities. Univ. Iagel. Acta Math. No. 45, 27-36 (2007)

7. A. Hefez. Irreducible Plane Curve Singularities. Sixth Worhshop at Sao Carlos. (2003), 1-120

8. A. Hefez, M.E. Hernandes, Analytic classification of plane branches up to multiplicity 4. J. Symb. Comput. 44, 626-634 (2009)

9. A. Hefez, M.E. Hernandes, M.F. Hernández Iglesias, On Polars of Plane Branches In: Cisneros-Molina J., Tráng Lê D., Oka M., Snoussi J. (eds) Singularities in Geometry, Topology, Foliations and Dynamics. Trends in Mathematics. Birkhäuser (2017), 135-153

10. A. Hefez; M.E. Hernandes; M.F. Hernández Iglesias, Plane branches with Newton non-degenerate polars. Int. J. Math. 29(1), 1850001 (2018)

11. M.F. Hernández Iglesias, Polar de um germe de curva irredutível plana. $\mathrm{PhD}$ thesis. Universidade Federal Fluminense, Brasil (2012)

12. M. Merle, Invariants polaires des courbes planes. Invent. Math. 41, 103-111 (1977)

13. M. Oka, Non-Degenerate Complete Intersection Singularity, Actualités Mathématiques. Hermann, Paris, viii+309 pp (1997)

14. B. Teissier, Varietés polaires. I. Invariants polaires des singularités d'hypersurfaces, Invent. Math. 40, 267-292 (1977)

15. O.Zariski, Characterization of plane algebroid curves whose module of differentials has maximum torsion. Proc. Nat. Acad. Sci. 56, 781-786 (1966)

16. O. Zariski, The moduli problem for plane branches, with an appendix by Bernard Teissier. University Lectures Series, Volume 39, AMS 2006, pp. 151

Publisher's Note Springer Nature remains neutral with regard to jurisdictional claims in published maps and institutional affiliations. 